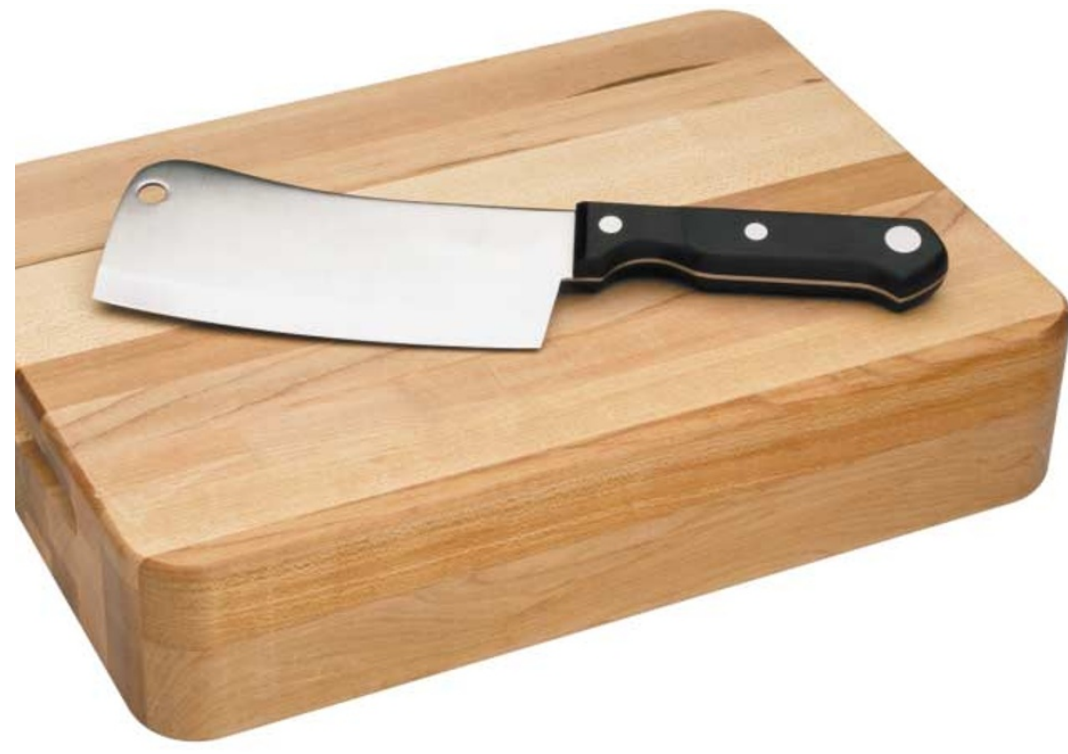

APOPTOSIS

\section{Cut to death}

The activity of caspase-family proteases is central to cell-death execution, although there is evidence for alternative caspase-independent pathways. Many proteins are cleaved by caspases during apoptosis, but whether a particular cut is actually required for cell death, or whether it is just a bystander event, is not always known. An elegant study by Jean Wang and colleagues published in Nature Cell Biology now shows that cleavage of retinoblastoma protein $(\mathrm{Rb})$ is required for apoptosis of certain tissues in response to specific death stimuli.

The cell-cycle regulator $\mathrm{Rb}$ is cleaved by caspases during apoptosis. The cleavage products are unstable and further degraded, resulting in the loss of Rb protein. $R b$-knockout mice show ectopic apoptosis, and fibroblasts derived from $R b$ deficient mice are hypersensitive to apoptosis induced by DNA damage and by tumour-necrosis factor (TNF)- $\alpha$. But, is cell death caused by the inability of $R b$-deficient cells to undergo cell-cycle arrest, or does $\mathrm{Rb}$ have a specific function in the inhibition of apoptosis, independent of its regulation of cell-cycle progression?

To address this issue, Wang and colleagues generated mice $(R b-M I)$ that contain a germline mutation in the caspase cleavage site in the $R b-1$ gene. Consistent with previous results in transfected fibroblasts, they found that expression of a caspase-resistant mutant of $\mathrm{Rb}$ results in protection from TNF- $\alpha$-induced cell death. More surprisingly, and in contrast to $R b$-deficient mice, $R b$ - $M I$ mice were as sensitive to radiation-induced apoptosis as wild-type mice.

In addition to the stimulus-specific effect of the $\mathrm{Rb}$ mutation, the authors also noticed cell-type-specific effects. Wild-type mice treated with bacterial lipopolysaccharide (LPS) undergo endotoxic shock and show massive cell death in the intestine and in the spleen. By contrast, the $R b-M I$ mutation protects intestinal cells, but not splenocytes, from LPS-induced cell death, and allows male, but not female, mice to survive.

So, this study indicates that cleavage and degradation of $\mathrm{Rb}$ is required for particular cell-death signalling pathways. The findings also raise questions regarding the mechanism of Rb's selective regulation of apoptosis. $\mathrm{Rb}$ has been reported to suppress the expression of cell-death regulatory genes, and it is also known that TNF- $\alpha$-induced killing does not require new gene expression. So, together, this indicates that Rb's function in apoptosis can be independent of gene transcription.

Valerie Ferrier, Associate Editor, Nature Cell Biology

\section{(0) References and links} ORIGINAL RESEARCH PAPER Chau, B. et al. Signal-dependent protection from apoptosis in mice expressing caspase-resistant $\mathrm{Rb}$. Nature Cell Biol. 4, 757-765 (2002)

\section{IN BRIEF}

\section{CELL SIGNALLING}

The ankyrin repeat protein Diversin recruits Casein kinase $\mid \varepsilon$ to the $\beta$-catenin degradation complex and acts in both canonical Wnt and Wnt/JNK signaling.

Schwarz-Romond, T. et al. Genes Dev. 16, 2073-2084 (2002)

This study describes a new vertebrate protein — Diversin — that is an essential component of the Wnt signalling pathway. Diversin acts as a molecular switch. It inhibits signals mediated by the canonical Wnt/ $\beta$-catenin pathway, which controls formation of the embryonic axis, and, at the same time, activates the Wnt/JNK signalling pathway, which establishes gastrulation movements in vertebrates.

\section{GENE EXPRESSION}

A recombinant RNA polymerase II-like enzyme capable of promoter-specific transcription.

Werner, F. \& Weinzierl, R. O. J. Mol. Cell (in the press)

So far, attempts to reconstitute active eukaryotic RNA polymerase (RNAP)-II from its individual subunits have failed. However, Werner and Weinzierl now report the successful in vitro assembly of the RNAPII-like, archaeal RNAP from purified recombinant subunits. The recombinant enzyme is fully active and, together with basal transcription factors TBP and TFB, is capable of promoter-specific transcription. This in vitro assembly approach also provided the authors with a unique opportunity to use mutagenesis studies to assess the functional contribution of individual subunits.

\section{CELL SIGNALLING}

A family of Rhomboid intramembrane proteases activates all Drosophila membrane-tethered EGF ligands.

Urban, S. et al. EMBO J. 21, 4277-4286 (2002)

Keren, a new ligand of the Drosophila epidermal growth factor receptor, undergoes two modes of cleavage.

Reich, A. \& Shilo, B. Z. EMBO J. 21, 4287-4296 (2002)

In the first of two papers that highlight the Drosophila epidermal growth factor (EGF) receptor (DER) pathway, Urban et al. investigated the processing of the DER ligands Spitz, Gurken and Keren. Rhomboid-1 is involved in the activation of Spitz by proteolytic cleavage, and analysis of four members of the Rhomboid family showed that Rhomboids 1-4 can proteolytically activate all three DER ligands. This common cleavage mechanism is distinct from that used to activate mammalian EGF-like ligands. In the second paper, Reich and Shilo describe the previously uncharacterized DER ligand Keren. Although Keren is processed as described above, proteolytic activation can also occur in a Rhomboidindependent manner, which highlights the complex regulation of this signalling pathway. 